Como citar este artigo:

Souza, NP; Valiatti, TB; Novais, VP; Romão, NF; Sobral, FOS; Avaliação De Cryptococcus Neoformans Em Excretas De Pombos (Columba Livia) No Perímetro Urbano Do Município De Ji-paraná, Rondônia, Brasil. Revista Saúde (Sta. Maria). 2018; 44 (3).

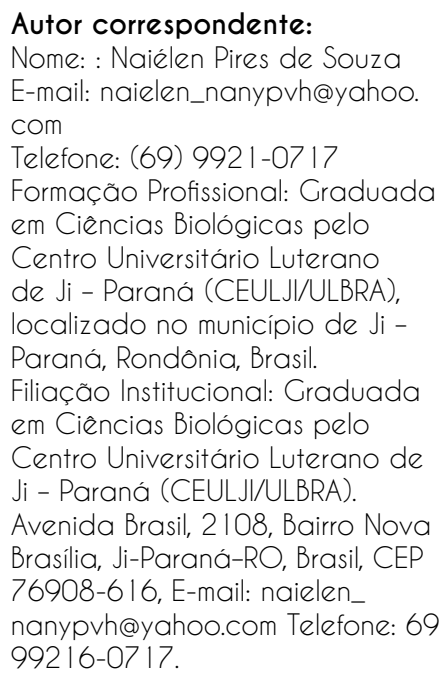

Data de Submissão:

30/03/2018

Data de aceite:

22/01/2019

Conflito de Interesse: Não há conflito de interesse

\section{Avaliação De Cryptococcus Neoformans Em Excretas De Pombos (Columba Livia) No Perímetro Urbano Do Município De Ji-paraná, Rondônia, Brasil}

\section{Evaluation of an experiment to determine whether cryptococcus neoformans is present in the excrement of pigeons (columba livia) in the city of ji-parana-ro}

\author{
Naiélen Pires de Souza, Tiago Barcelos Valiatti, Valéria Pinheiro Novais, Natália \\ Faria Romão, Fabiana De Oliveira Solla Sobral
}

\section{RESUMO}

Os fungos do gênero Cryptococcus são os causadores da Criptococose em humanos e animais, especialmente Cryptococcus neoformans e Cryptococcus gattii. São fungos encontrados em diversas fontes ambientais, dentre elas as fezes dos pombos. Esse trabalho objetivou analisar se há a presença deste fungo na cidade de Ji-paraná nas fezes dos pombos. A escolha dos pontos de coleta baseou-se na alta população de pombos povoando o local, onde foram colhidas 50 amostras em 10 lugares diferentes. A metodologia usada foi descrita por Contin13, sendo realizada a inoculação em ágar Sabouraud, confirmação de cápsula através da microscopia e prova da uréase. Resultados mostraram que das 50 amostras, $26 \%$ foram positivas para o Cryptococcus neoformans, confirmando sua ocorrência na cidade de Ji-paraná-RO. Este estudo é inédito na cidade, sendo assim, há a necessidade de mais estudos com este enfoque. Apesar disso, é importante evitar o contato com pombos e suas excretas.

Descritores: criptococose, Cryptococcus neoformans, fungos, Columbia livia.

\section{ABSTRACT}

Fungi from the genre Cryptococcus are the causative agents of Criptococose in humans and animals, especially Cryptococcus neoformans and Cryptococcus gattii. These are fungi found in a variety of environmental sources including pigeon excrement. This study's goal is to ascertain whether this fungus is present in the excrement of pigeons from the city of Ji-Paraná or not. The choice of sample collection points focused on areas with a high population of pigeons. 50 samples were collected in 10 different spots. The methodology described by Contin 13 was applied in this study. Inoculation by Sabouraud agar, capsule confirmation through microscopy, and a urease test were carried out in the process. The results have shown that $26 \%$ out of the 50 samples tested positive for the presence of Cryptococcus neoformans. This confirms the presence of this fungus in the excrement of the pigeons of Ji-Paraná-RO. This study is the first of its kind in this city. It highlights the need for more similar studies in the future. Nevertheless, it is obviously important to avoid contact with pigeons and their excreta for the time being.

Descriptors: cryptococcosis; Cryptococcus, neoformans, fungi, Columbia livia. 


\section{INTRODUÇÃO}

Infecções oportunistas são comuns em pacientes debilitados e imunocompetentes ${ }^{1}$, dentre as quais os fungos assumem um papel importante devido à sua patogenicidade e sua rápida disseminação e propagação². Em meio as doenças fungicas, a criptococose se caracteriza como uma micose sistêmica com altos índices de morbidade, principalmente em pacientes imunocomprometidos, acometendo primariamente os pulmões, apresentando tropismo para o sistema nervoso central ${ }^{3,5}$.

Essa patologia tem como agente causador o gênero Cryptococcus, especialmente as espécies Cryptococcus neoformans e Cryptococcus gattii ${ }^{6}$, diferentes entre si em aspectos ecológicos, genéticos, bioquímicos e antigênicos ${ }^{7}$.

O Cryptococcus neoformans possui duas fases de vida, uma fase assexuada e outra fase sexuada, sendo a fase sexuada a forma leveduriforme, que é a encontrada em homens e animais infectados ${ }^{8}$. Adicionalmente, a espécie Cryptococcus neoformans, se divide em três variedades e cinco sorotipos, sendo o sorotipo (A) pertencente a variedade grubii, sorotipos (AD e D), pertencentes a variedade neoformans e sorotipos $(\mathrm{B}$ e $\mathrm{C})$ à variedade gattii ${ }^{\text {. }}$.

Os fatores que induzem a patogenicidade estão divididos em dois grupos: um relacionado às características de estabelecimento da infecção, e o outro relacionado aos fatores de virulência afetando o grau de patogenia ${ }^{10}$. A doença é adquirida através da inalação de propágulos infectantes, que são comumente encontrados nas fezes de algumas aves incluindo passeriformes e columbiformes que incluem os pombos (Columba livia) ${ }^{11}$, embora alguns autores ${ }^{12,13}$ considerem pouco provável que o pombo seja sua principal fonte de disseminação na natureza.

Contudo, as fezes destas aves são ricas em nitrogênio, creatinina e uréia, oferecendo condições adequadas para a proliferação dos propágulos fungicos ${ }^{10,11}$, deste modo, os pombos carregam o fungo em suas penas, patas, cloaca e bicos e servem de carreadoras, disseminando o fungo para outros ambientes ${ }^{14}$.

Segundo Faria et al..$^{15}$, o ecossistema urbano tem se tornado propício a permanência destas aves, pois em tal ambiente estes animais encontram abrigo e alimento com facilidade, proporcionando acúmulo de excretas, nas quais se alojam os propágulos infectantes, podendo permanecer viáveis para contágio durante dois anos, fazendo desta patologia uma doença de caráter cosmopolita ${ }^{10}$.

As complicações dessa infecção incluem, meningoencefalite, sendo sua evolução grave e fatal. Essa patologia pode ser acompanhada ou não de lesão pulmonar, focos secundários nos rins, pele e ossos ${ }^{10,11}$. A infecção ocorre primariamente no pulmão, a partir de onde ocorre a disseminação para outros órgãos causando lesões generalizadas ou sistêmicas. Tecidos como olhos, ossos e articulações podem ser acometidos, mas em regra, o Sistema Nervoso Central (SNC) é a parte mais atingida pela doença ${ }^{11,14}$.

$O$ diagnóstico da doença é confirmado através do isolamento do fungo em secreções biológicas como líquor, sangue e urina, podendo ser realizados exames diretos ou culturas. Algumas drogas que são usadas no tratamento incluem Anfotericina $B$, Fluconazol, onde o tratamento varia de 6 a 12 meses conforme estado clínico do paciente ${ }^{16}$.

O objetivo do presente estudo foi verificar se nas fezes dos pombos ocorre o fungo Cryptococcus neoformans que causa a criptococose em humanos e animais, no município de Ji-Paraná, Rondônia.

\section{MATERIAIS E MÉTODOS}

Para elaboração do presente estudo, foram selecionados 10 diferentes pontos do município, onde, em cada, coletou-se 5 amostras, perfazendo um total de 50 amostras. Após a coleta, as amostras foram levadas ao laboratório de Microbiologia, do Centro 
Universitário Luterano de Ji-Paraná (CEULJI/ULBRA), onde se seguiu com as análises.

As amostras foram trituradas e homogeneizadas em gral com pistilo de porcelana esterilizado, sendo que, em seguida 5 gramas do material foi suspenso em $5 \mathrm{~mL}$ de cloreto de sódio a 0,9\% estéril e homogeneizado em vórtex por três minutos e deixado repousar por 30 minutos em temperatura ambiente. Posteriormente, retirou-se $0,1 \mathrm{~mL}$ do sobrenadante e semeou-se em placas de petri contendo Agar Sabouraud dextrose $2 \%$, sendo essas em seguida incubadas a $30^{\circ} \mathrm{C}$ por sete dias conforme metodologia utilizada e descrita por Contin ${ }^{17}$. Após esse período, realizou-se uma análise macroscópicas e microscópicas das colônias características do fungo em questão, as quais foram repicadas por estriamento em placas de petri contendo Agar Sabouraud dextrose $2 \%$ para isolamento e identificação confirmatória por microscopia e prova de uréase. Como o fungo apresenta capacidade de hidrolisar uréia, foi então verificada a viragem da cor indicadora amarelo inicial do meio, para cor rosa ou vermelho, sendo então estas positivas para o fungo Cryptococcus neoformans ${ }^{17}$.

\section{RESULTADOS E DISCUSSÃO}

O presente estudo é o primeiro a ser desenvolvido no município de Ji-Paraná, RO, contribuindo assim, com dados inéditos para a cidade, que, se demonstraram preocupantes, visto que houve a positividade para $C$. neoformans. Entretanto, este não é o primeiro estudo a nível estadual, visto que, Côrrea et al. ${ }^{8}$, em estudo anterior no município de Cacoal, ao analisaram fezes de pombos de 2 pontos do município, também verificaram positividade para $C$. neoformans.

Os resultados encontrados, demonstraram que das 50 amostras analisadas, $26 \%$ foram positivas para C. neoformans, conforme demonstrado na figura 1.

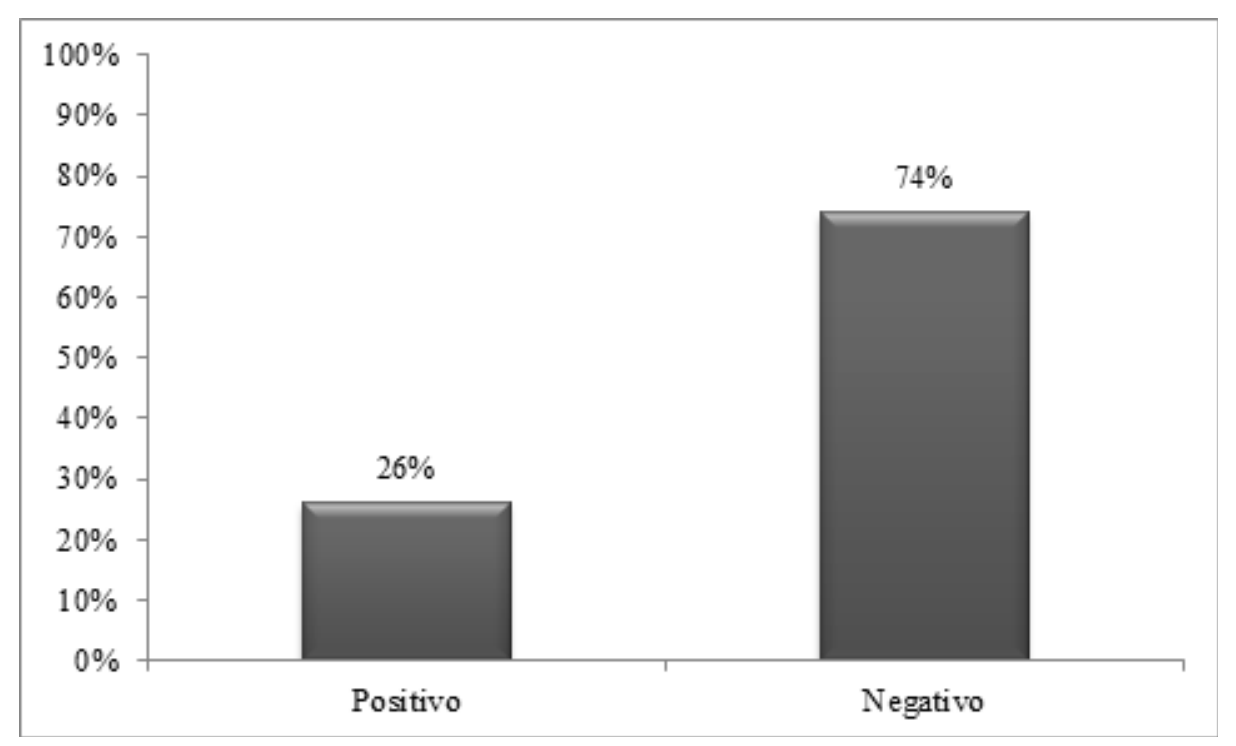

Figura 1. Resultados observados com relação as análises de C. neoformans em amostras de excretas de pombos.

Estudos disponíveis na literatura demonstram a presença de $C$. neoformans em outras partes do pais, tais como, Menezes et $a .^{18}$ que ao analisar 195 amostras de fezes de pombos em Lages, SC, verificaram a presença do fungo aqui em questão em $7,69 \%$ das amostras. Já, Contin et al..$^{17}$ observou uma prevalência de C. neoformans em $100 \%$ das amostras de fezes coletadas no município de Caratinga, Minas Gerais. Ainda, outros estudos desenvolvidos em Pelotas - RS ${ }^{15}$ e São José dos Campos - SP ${ }^{19}$ também relataram a presença de $C$. neofarmans.

Os resultados normalmente variam de uma região para outra, fato este, devido a uma série de fatores que influenciam 
diretamente esses resultados. Dentre esses fatores, destacam-se as condições meteorológicas do local de estudo, visto que, a temperatura exerce influencia na proliferação de microrganismos, sendo que as regiões quentes e úmidas são propícias para 0 crescimento fúngico. Ainda, a época de coleta, quantidade, e tipo de amostra (secas ou úmidas) também interferem no resultado final ${ }^{20-23}$.

A presença de $C$. neoformans na maioria das vezes está nas fezes de $C$. livia, no entanto é possível encontrar na literatura sua presença em excretas de outros tipos de aves como, Columba sp. (pombo rabo de leque), Melopsittacus undulatus (periquito australiano), Nymphicus hollandicus (calopsita), Taeniopigya guttata (mandarim), Serinus canaria (canário belga) e Agapornis $s p$. (agapore) ${ }^{24}$. Tal fato se justifica devido as fezes dessas outras aves também apresentarem condições propícias para 0 desenvolvimento e reprodução desse fungo ${ }^{25}$.

Estudo feito por Lugarini ${ }^{26}$, no estado do Paraná, evidenciou pela primeira vez, o isolamento de $C$. neoformans em excretas de Passeriformes e Psitaciformes, e demonstram a importância dessas e de outras aves como reservatórios de Cryptococcus no ambiente, e consequentemente na epidemiologia da Criptococose.

Dentre as diversas características que contribuem para a virulência do $C$. neofarmans, a sua cápsula polissacarídica é extremamente importante, pois a mesma atua como um escudo, o protegendo das adversidades do ambiente ${ }^{27}$. Com relação ao hospedeiro, a cápsula age interferindo na resposta imunológica28-31.

Na figura 2, encontra-se o resultados observados, estratificados por locais de coleta, onde, veerifica-se maior positividade no ponto 1. Destaca-se resultados satisfatórios nos pontos 5, 6,8 e 10, visto que não houve presensa de $C$. neoformans.

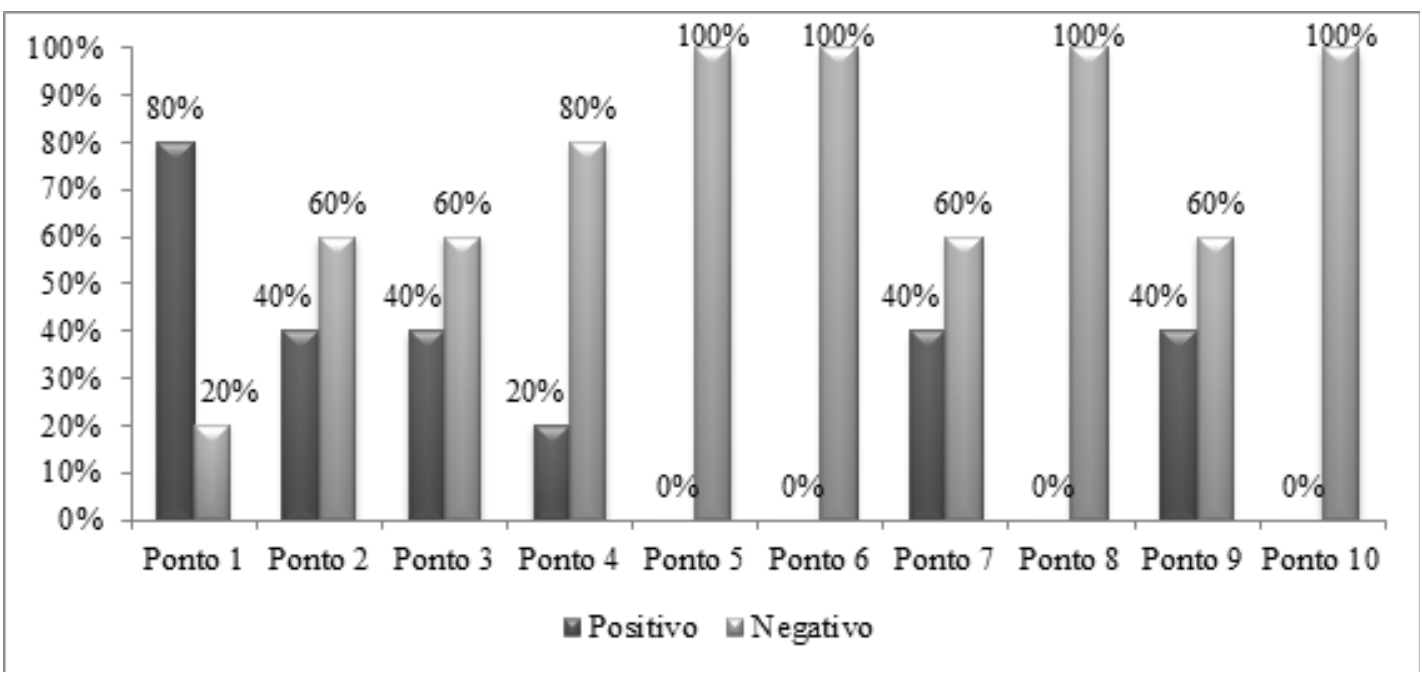

Figura 2. Resultados observados com relação as análises de C. neoformans em amostras de excretas de pombos estratificados por local de coleta.

Conforme observado, constatou-se que o ponto 1 foi o que apresentou maior índice de contaminação, tal dado se justifica devido às condições do local, visto que o mesmo é em uma localidade mais afastada que não recebe limpeza frequentemente como os outros pontos de coleta. Colombo et al..$^{21}$ destaca que a realização de limpeza e desinfecção onde há acúmulo de fezes de aves é uma importante medida para o controle epidemiológico.

Ficher e $\operatorname{Cook}^{32}$ destacam que ao umedecer as fezes, uma maior atividade e multiplicação de bactérias será registrada, que consequentemente irá promover uma alcalinização do substrado juntamente com uma competição alimentar com a levedura, ocorrendo assim a inibição do crescimento de C. neoformans. 
Os dados revelados pelo presente estudo demonstra existir um certo risco para população, pois demonstram que os pombos estão trazendo para o perímetro urbano do município o $C$. neoformans. $O$ aumento da população de pombos tem sido caracterizado como um problema ambiental e de saúde pública, visto que estas aves possuem preferência de habitar-se em locais de acesso difícil, permitindo assim que o fungo permaneça por vários anos ${ }^{33-35}$. Vale salientar que os pombos compõem a população de animais silvestres e, portanto, seu controle deve ser feito somente por pessoas autorizadas.

\section{CONCLUSÃO}

Através deste estudo, foi constatado que ocorre a contaminação por c. neoformans em 6 dos locais coletados, confirmando sua ocorrência na cidade de Ji-paraná, demonstrando a necessidade de desenvolvimento de medidas preventivas por parte dos órgãos competentes, visto a gravidade que estes podem representar para sociedade.

\section{REFERÊNCIAS}

1. Fernandes OFL, Costa TR, Costa MR, Soares AJ, Pereira AJSC, Silva MRR. Cryptococcus neoformans in patients with AIDS. Rev Soc Bras Med Trop. 2000; 33(1):75-78.

2. Wicles BL, et al. Dimorphism and haploid fruiting in Cryptococcus neoformans association with the a-matin type. Proceeding of the National Academy of Science. 1996; 93(4): 7327-31.

3. Lopes RCO. Criptococose: aspectos epidemiológicos e patológicos em pacientes com HIV - Revisão Bibliográfica. Brasília: Universidade Católica de Brasília; 2014.

4. Lima CT, Klafke GB, Xavier MO. Cryptococcus spp. em excretas de Columba livia (pombos domésticos) provenientes de um hospital universitário no Sul do Brasil. Arq. Inst. Biol. 2015; 82: 1-4.

5. Favalessa, OC; Ribeiro LC.; Tadano T, Fontes CJF, Dias FB, Coelho BPA, et al. First description of phenotypic profile and in vitro drug susceptibility of Cryptococcus spp yeast isolated from HIV positive and HIV- negative patients in State of Mato Grosso. Rev. Soc Bras Med Trop. 2009; 42(6): 661-665.

6. Brasil. Ministério da Saúde. Vigilância epidemiológica da criptococose. Unidade de vigilância das doenças de transmissão respiratória e imunupreveníveis. Brasília. Brasil. 2012.

7. Reolon A, Perez LRR, Mezzari A. Prevalência de Cryptococcus neoformans nos pombos urbanos da Cidade de Porto Alegre, Rio Grande do Sul. Jor Bras de Pat e Med Lab. 2004; 40(5):.293-298.

8. Corrêa EA, Naconechny F, Casagrande PL. Presença de cryptococcus neoformans em excretas de Columba.Sp. Na cidade de Cacoal, Rondônia, Brasil. Revi Igapó - IFAM. 2011;:5(1).

9. Silva MAP, Gagliani LH. Diagnóstico e prevalência da meningite criptococócica em pacientes portadores da Síndrome da Imunodeficiência Adquirida - SIDA. Ver UNILUS Ensino e Pesquisa. 2014.; 11(22).

10. Takahara DT. Isolamento e identificação de Cryptococcus neoformans a partir de excretas de pombos provenientes de locais públicos e residenciais de Cuiabá e Várzea Grande - MT [Dissertação]. Cuiabá: Universidade Federal de Mato Grosso; 2013. 
11. Scain G. Prevalência de Cryptococcus neoformans em fezes de pombos (Columba livia) nas praças públicas da cidade de Lages, Santa Catarina [Dissertação]. Lages: Universidade do Extremo Sul Catarinense - UNESC; 2011.

12. Littman LM, Borok R. Relation of the pigeon to cryptococcosis: Natural carrier state, heat resistance and survival of Cryptococcus neoformans. Mycopathol Mycol Appl. 1968; 35: 922-933.

13. Abou-Gabal M, and Atia M. Study of the role of pigeons in the dissemination of Cryptococcus neoformans in nature. Sabouraudia. 1978; 16: 63-68.

14. Egres CC. Isolamento ambiental de Cryptococcus spp em hospital de Porto Alegre - RS [Dissertação]. Porto Alegre: Universidade Federal do Rio Grande do Sul; 2010.

15. Faria RO, Nascente SP, Meinerz MAR, Cleff BM, Antunes TA, Silveira SE, et al. Ocorrência de Cryptococcus neoformans em excretas de pombos na cidade de Pelotas, Estado do Rio Grande do Sul. Rev Soc Bras Med Trop. 2010; .43: 198-200.

16. Queiroz JPA. Criptococcose - uma revisão bibliográfica. Acta Veterinaria Basilica. 2008; v:(2), p 32-38.

17. Contin TJ, Quaresma SG, Silva FE. Ocorrência de cryptococcus neoformans em fezes de pombos na cidade de Caratinga, Minas Gerais, Brasil. Rev Med Min Ger. 2009;.21: 19-24.

18. Menezes T, Scain G, Quadros, RM, Miletti LC, Souza AL, Miguel RL, Marques SMT. Cryptococcus spp. em excretas de pombos (Columba livia) de áreas públicas de Lages, Santa Catarina. Science and Aninal Health.2014; 2(2): 102-114.

19. Ribeiro MFP, Silva AM, Fernandes WS, Mello MM. Isolamento de Cryptococcus neofarmans em fezes de pombos (Columba livia) em praças públicas de São José dos Campos - SP. J Health Sci Inst. 2017; 35(1)::23-27.

20. Reolon A, Perez LRR, Mezzari A. Prevalência de Cryptococcus neoformans nos pombos urbanos da cidade de Porto Alegre, Rio Grande do Sul. J Bras Patol Med Lab. 2004;40(5):293-8.

21. Colombo G, Táparo CV, Araújo Júnior EC, Makatu MY, Santos FS, Marinho M. Caracterização bioquímica e molecular de Cryptococcusspp. isolados de excretas ambientais de pombos (Columbalivia doméstica). Arq Bras Med Vet Zootec. 2015;67(6):1639-45.

22. Araújo Júnior EC, Táparo CV, Uchida CY, Marinho M. Cryptococcus: isolamento ambiental e caracterização bioquímica. Arq Bras Med Vet. Zootec. 2015;67(4):1003-8.

23. Soares MCB, Paula CR, Dias ALT, Caseiro MM, Costa SOP. Environmental strains of Cryptococcus neoformans variety grubii in the city of Santos, SP, Brazil. Rev Inst Med Trop. 2004;47(1): 31-6.

24. Filiú WFO, Wanke B, Agüena SM, et al. Cativeiro de aves como fonte de Cryptococcus neoformans na cidade de Campo Grande, Mato Grosso do Sul, Brasil. Rev. Soc. Bras. Med. Trop. 2002; 35: 591-595.

25. Levitz SM. The ecology of Cryptococcus neoformans and the epidemiology of cryptococcosis. Rev Infect Dis 1991; 13:11631169.

26. Lugarini C. Isolamento de Cryptococcus neoformans a partir de excretas Passeriformes e psittaciformes no estado do Paraná. [Tese de Mestrado] Curitiba. Universidade Federal do Paraná; 2007.

27. Steenbergen JN, Nosanchuk JD, Stephanie D, Casadevall A. Cryptococcus neoformans Virulence Is Enhanced after Growth in the Genetically Malleable Host Dictyostelium discoideum. Infect Immun, 2003;71: 4862-72. 
28. Vecchiarelli A, Pietrella D, Lupo P, Bistoni F, Mcfadden DC, Casadevall A. The polysaccharide capsule of Cryptococcus neoformans interferes with human dendritic cell maturation and activation. J Leukoc Biol. 2003;74:370-8.

29. Voelz, K; May RC. Cryptococcal interactions with the host immune system. Eukaryot Cell. 2010; 9:835-46.

30 Zaragoza, O. Multiple Disguises for the Same Party: The Concepts of Morphogenesis and Phenotypic Variations in Cryptococcus neoformans. Front Microbiol, 2011; 2: 181.

31. O' Meara TR, Alspaugh JA. The Cryptococcus neoformans Capsule : a Sword and a Shield. Clin Microbiol Rev. 2012; 25: $387-408$.

32. Fisher F, Cook NB. Micologia: fundamentos e diagnóstico. Rio de Janeiro: Revinter, 2001. 337p.

33. Cermeño JR, Hernández I, Cabello I, et al. Cryptococcus neoformans and Histoplasma capsulatum in dove's (Columbia livia) excreta in Bolívar State, Venezuela. Revista Latinoamericana de Microbiologia. 2006; 48(1): 6-9.

34. Rosario I, Acosta B, Colom F. La Paloma y otras aves como reservorio de Cryptococcus spp. Revista Iberoamericana de Micologia. 2008; (25): S13-S18.

35. Takahara DT, Lazéra MS, Wanke B, et al. Primeiro registro de Cryptococcus neoformans em excretas de pombos provenientes de locais públicos e residenciais de área metropolitana de Cuiabá, Estado do Mato Grosso, Brasil. Rev do Inst de Med Trop. 2013; 55(6): 371-376. 